\title{
Radial Two Weight Inequality for Maximal Bergman Projection Induced by a Regular Weight
}

\section{Taneli Korhonen $^{1}$ (D) . José Ángel Peláez ${ }^{2}$ Jouni Rättyä}

Received: 27 May 2019 / Accepted: 10 February 2020 / Published online: 29 August 2020

(C) The Author(s) 2020

\section{Abstract}

It is shown in quantitative terms that the maximal Bergman projection

$$
P_{\omega}^{+}(f)(z)=\int_{\mathbb{D}} f(\zeta)\left|B_{z}^{\omega}(\zeta)\right| \omega(\zeta) d A(\zeta),
$$

is bounded from $L_{v}^{p}$ to $L_{\eta}^{p}$ if and only if

$$
\sup _{0<r<1}\left(\int_{0}^{r} \frac{\eta(s)}{\left(\int_{s}^{1} \omega(t) d t\right)^{p}} d s+1\right)^{\frac{1}{p}}\left(\int_{r}^{1}\left(\frac{\omega(s)}{v(s)^{\frac{1}{p}}}\right)^{p^{\prime}} d s\right)^{\frac{1}{p^{\prime}}}<\infty
$$

provided $\omega, v, \eta$ are radial regular weights. A radial weight $\sigma$ is regular if it satisfies $\sigma(r)=\int_{r}^{1} \sigma(t) d t /(1-r)$ for all $0 \leqslant r<1$. It is also shown that under an appropriate additional hypothesis involving $\omega$ and $\eta$, the Bergman projection $P_{\omega}$ and $P_{\omega}^{+}$are simultaneously bounded.

Keywords Bergman projection · Bergman space $\cdot$ Regular weight $\cdot$ Two weight inequality

Mathematics Subject Classification (2010) $30 \mathrm{H} 20 \cdot 46 \mathrm{E} 30 \cdot 47$

Dedicated to Fernando Pérez-González on the occasion of his retirement

This research was supported in part by Ministerio de Economía y Competitivivad, Spain, projects PGC2018-096166-B-100 and MTM2017-90584-REDT; La Junta de Andalucía, project FQM210; Academy of Finland project no. 268009.

\footnotetext{
Taneli Korhonen

taneli.korhonen@uef.fi

José Ángel Peláez

japelaez@uma.es

Jouni Rättyä

jouni.rattya@uef.fi
}

1 University of Eastern Finland, P.O.Box 111, 80101 Joensuu, Finland

2 Departamento de Análisis Matemático, Universidad de Málaga, Campus de Teatinos, 29071 Málaga, Spain 


\section{Introduction and Main Results}

A function $\omega: \mathbb{D} \rightarrow[0, \infty)$, integrable over the unit disc $\mathbb{D}$, is called a weight. It is radial if $\omega(z)=\omega(|z|)$ for all $z \in \mathbb{D}$. For $0<p<\infty$ and a weight $\omega$, the Lebesgue space $L_{\omega}^{p}$ consists of complex-valued measurable functions $f$ in $\mathbb{D}$ such that

$$
\|f\|_{L_{\omega}^{p}}=\left(\int_{\mathbb{D}}|f(z)|^{p} \omega(z) d A(z)\right)^{\frac{1}{p}}<\infty,
$$

where $d A(z)=\frac{d x d y}{\pi}$ denotes the element of the normalized Lebesgue area measure on $\mathbb{D}$. The weighted Bergman space $A_{\omega}^{p}$ is the space of analytic functions in $L_{\omega}^{p}$, and is equipped with the corresponding $L_{\omega}^{p}$-norm. If the norm convergence in the Hilbert space $A_{\omega}^{2}$ implies the uniform convergence on compact subsets of $\mathbb{D}$, the point evaluations are bounded linear functionals on $A_{\omega}^{2}$. Therefore there exist reproducing Bergman kernels $B_{z}^{\omega} \in A_{\omega}^{2}$ such that

$$
f(z)=\left\langle f, B_{z}^{\omega}\right\rangle_{A_{\omega}^{2}}=\int_{\mathbb{D}} f(\zeta) \overline{B_{z}^{\omega}(\zeta)} \omega(\zeta) d A(\zeta), \quad z \in \mathbb{D}, \quad f \in A_{\omega}^{2} .
$$

The Hilbert space $A_{\omega}^{2}$ is a closed subspace of $L_{\omega}^{2}$, and hence the orthogonal projection from $L_{\omega}^{2}$ to $A_{\omega}^{2}$ is given by

$$
P_{\omega}(f)(z)=\int_{\mathbb{D}} f(\zeta) \overline{B_{z}^{\omega}(\zeta)} \omega(\zeta) d A(\zeta), \quad z \in \mathbb{D} .
$$

The operator $P_{\omega}$ is the Bergman projection.

In this paper we will characterize the radial two-weight inequality

$$
\left\|P_{\omega}^{+}(f)\right\|_{L_{\eta}^{p}} \leqslant C\|f\|_{L_{v}^{p}}, \quad f \in L_{v}^{p},
$$

for the maximal Bergman projection $P_{\omega}^{+}(f)(z)=\int_{\mathbb{D}} f(\zeta)\left|B_{z}^{\omega}(\zeta)\right| \omega(\zeta) d A(\zeta)$ under certain smoothness requirements on the three radial weights involved. The question of when (1.1) is satisfied is an open problem even in the very particular case $\omega=v=\eta$ if no preliminary hypotheses is imposed on the radial weight.

Two weight inequalities for classical operators have attracted a considerable amount of attention in Complex and Harmonic Analysis, and are closely connected to other interesting questions in the area $[2,5-7,10,11]$. The most commonly known result on Bergman projection is due to Bekollé and Bonami [3, 4], and concerns the case when $v=\eta$ is an arbitrary weight and the inducing weight $\omega$ is standard, that is, of the form $\omega(z)=\left(1-|z|^{2}\right)^{\alpha}$ for some $\alpha>-1$; see $[1,11,13]$ for recent extensions of this result. In this classical case, the Bergman reproducing kernel $B_{z}^{\omega}(\zeta)$ is given by the neat formula $(1-\bar{z} \zeta)^{-(2+\alpha)}$. However, for a general radial weight $\omega$ such explicit formulas for the kernels do not necessarily exist, and that is one of the main obstacles in tackling (1.1). Moreover, kernels induced by radial weights may have zeros, and that of course does not make things any easier. Nonetheless, (1.1) has been recently characterized in the particular case $v=\eta$ provided $\omega$ and $v$ are regular weights [10].

For a radial weight $\omega$, we assume throughout the paper that $\widehat{\omega}(z)=\int_{|z|}^{1} \omega(s) d s>0$ for all $z \in \mathbb{D}$, for otherwise the Bergman space $A_{\omega}^{p}$ would contain all analytic functions in $\mathbb{D}$. A radial weight $\omega$ belongs to the class $\hat{\mathcal{D}}$ if there exists a constant $C=C(\omega)>1$ such 
that $\widehat{\omega}(r) \leqslant C \widehat{\omega}\left(\frac{1+r}{2}\right)$ for all $0 \leqslant r<1$. Moreover, if there exist $K=K(\omega)>1$ and $C=C(\omega)>1$ such that

$$
\widehat{\omega}(r) \geqslant C \widehat{\omega}\left(1-\frac{1-r}{K}\right), \quad 0 \leqslant r<1,
$$

then we write $\omega \in \check{\mathcal{D}}$. The intersection $\widehat{\mathcal{D}} \cap \breve{\mathcal{D}}$ is denoted by $\mathcal{D}$. A radial weight $\omega$ is regular if $\widehat{\omega}(r) \asymp \omega(r)(1-r)$ for all $0 \leqslant r<1$. The class of regular weights is denoted by $\mathcal{R}$, and $\mathcal{R} \subsetneq \mathcal{D}$. For basic properties of these classes of weights and more, see [8, 9, 12] and the references therein.

The main result of this study is the following theorem, which provides a quantitative description of the boundedness of $P_{\omega}^{+}: L_{\nu}^{p} \rightarrow L_{\eta}^{p}$ in terms of a Muckenhoupt-type condition related to weighted Hardy operators.

Theorem 1 Let $1<p<\infty, \omega, v \in \mathcal{R}$ and $\eta \in \check{\mathcal{D}}$. Then $P_{\omega}^{+}: L_{v}^{p} \rightarrow L_{\eta}^{p}$ is bounded if and only if

$$
M_{p}(\omega, v, \eta)=\sup _{0<r<1}\left(\int_{0}^{r} \frac{\eta(s)}{\widehat{\omega}(s)^{p}} d s+1\right)^{\frac{1}{p}}\left(\int_{r}^{1}\left(\frac{\omega(s)}{v(s)^{\frac{1}{p}}}\right)^{p^{\prime}} d s\right)^{\frac{1}{p^{\prime}}}<\infty .
$$

Moreover, $\left\|P_{\omega}^{+}\right\|_{L_{v}^{p} \rightarrow L_{\eta}^{p}} \asymp M_{p}(\omega, v, \eta)$.

The key tools in the proof of Theorem 1 are the precise estimates for the $L^{p}$-means and $A_{v}^{p}$-norms of the Bergman kernel $B_{z}^{\omega}$ obtained in [10, Theorem 1]. A special case of the said result is repeatedly used in the proof and it is stated for further reference as Theorem $\mathrm{A}$ below. For a function $f$ analytic in $\mathbb{D}$ and $0<r<1$, write

$$
M_{p}(r, f)=\left(\frac{1}{2 \pi} \int_{0}^{2 \pi}\left|f\left(r e^{i t}\right)\right|^{p} d t\right)^{\frac{1}{p}}, \quad 0<p<\infty .
$$

Theorem A Let $0<p<\infty$ and $\omega, v \in \widehat{\mathcal{D}}$. Then the following assertions hold:

(i) $M_{p}^{p}\left(r, B_{a}^{\omega}\right)=\int_{0}^{|a| r} \frac{d t}{\widehat{\omega}(t)^{p}(1-t)^{p}}, \quad r,|a| \rightarrow 1^{-}$;

(ii) $\left\|B_{a}^{\omega}\right\|_{A_{v}^{p}}^{p}=\int_{0}^{|a|} \frac{\widehat{v}(t)}{\widehat{\omega}(t)^{p}(1-t)^{p}} d t, \quad|a| \rightarrow 1^{-}$.

It is worth noticing that the upper estimate

$$
\left\|B_{a}^{\omega}\right\|_{A_{v}^{p}}^{p} \lesssim \int_{0}^{|a|} \frac{\widehat{v}(t)}{\widehat{\omega}(t)^{p}(1-t)^{p}} d t, \quad|a| \rightarrow 1^{-},
$$

holds for $\omega \in \widehat{\mathcal{D}}$ and any radial weight $v$ [10, p. 106].

The argument used to establish the one weight inequality [10, Theorem 3] for regular weights does not carry over as such to the two weight case. The proof of the sufficiency in Theorem 1 is much more involved due to the presence of the second weight $\eta$.

The operators $P_{\omega}$ and $P_{\omega}^{+}$are simultaneously bounded under a natural additional hypothesis. This is the content of the other main result of this study. 
Theorem 2 Let $1<p<\infty, \omega, v \in \mathcal{R}$ and $\eta \in \mathcal{D}$ such that

$$
\sup _{0<r<1}\left(\int_{0}^{r}\left(\frac{\omega(s)}{\eta(s)^{\frac{1}{p}}}\right)^{p^{\prime}}\left(\int_{s}^{1}\left(\frac{\omega(t)}{v(t)^{\frac{1}{p}}}\right)^{p^{\prime}} d t\right)^{-p} d s+1\right)^{\frac{1}{p}}\left(\int_{r}^{1}\left(\frac{\omega(s)}{v(s)^{\frac{1}{p}}}\right)^{p^{\prime}} d s\right)^{\frac{1}{p^{\prime}}}<\infty .
$$

Then the following statements are equivalent:

(i) $P_{\omega}^{+}: L_{v}^{p} \rightarrow L_{\eta}^{p}$ is bounded;

(ii) $\quad P_{\omega}: L_{v}^{p} \rightarrow L_{\eta}^{p}$ is bounded;

$$
N_{p}(\omega, v, \eta)=\sup _{0<r<1} \frac{\hat{\eta}(r) \frac{1}{p}}{\widehat{\omega}(r)}\left(\int_{r}^{1}\left(\frac{\omega(s)}{v(s)^{\frac{1}{p}}}\right)^{p^{\prime}} d s\right)^{\frac{1}{p^{\prime}}}<\infty
$$

Theorem 2 is a generalization of [10, Theorem 3] because the hypothesis (1.5) is satisfied for $v=\eta$. Although, the conditions $N_{p}(\omega, v, \eta)<\infty$ and $M_{p}(\omega, v, \eta)<\infty$ are equivalent for many weights, for example the standard weights have this property, the condition $N_{p}(\omega, v, \eta)<\infty$ may be essentially weaker than $M_{p}(\omega, v, \eta)<\infty$ under the hypothesis of Theorem 1. Indeed, if we pick up an arbitrary $\omega \in \mathcal{R}$, and define $v(s)=$ $\omega(s) \widehat{\omega}(s)^{\frac{p}{p^{\prime}}}\left(\log \frac{e}{1-s}\right)^{2 \frac{p}{p^{\prime}}}$ and $\eta(s)=\omega(s) \widehat{\omega}(s)^{\frac{p}{p^{\prime}}}\left(\log \frac{e}{1-s}\right)^{\frac{p}{p^{\prime}}}$, then $\nu, \eta \in \mathcal{R}$ and one can show by using Lemmas B and $\mathrm{C}$ below that $N_{p}(\omega, v, \eta)<\infty$ but $M_{p}(\omega, v, \eta)=\infty$. Now of course (1.5) fails for these choices of weights.

It is readily seen that the methods used to prove Theorems 1 and 2 carry over to the case $p=1$. In fact, the proof in this case turns out much more simple for obvious reasons. To be precise, one can show that in the case $p=1$ the operators $P_{\omega}$ and $P_{\omega}^{+}$are simultaneously bounded, and the uniform boundedness of the quantity

$$
\frac{\omega(r)}{v(r)} \int_{0}^{1} \frac{\eta(t)}{\widehat{\omega}(t r)} d t
$$

is the characterizing condition.

Throughout the paper $\frac{1}{p}+\frac{1}{p^{\prime}}=1$ for $1<p<\infty$. Further, the letter $C=C(\cdot)$ will denote an absolute constant whose value depends on the parameters indicated in the parenthesis, and may change from one occurrence to another. We will use the notation $a \lesssim b$ if there exists a constant $C=C(\cdot)>0$ such that $a \leqslant C b$, and $a \gtrsim b$ is understood in an analogous manner. In particular, if $a \lesssim b$ and $a \gtrsim b$, then we will write $a \asymp b$.

\section{Proof of Theorem 1}

Throughout the proofs we will repeatedly use several basic properties of weights in the classes $\widehat{\mathcal{D}}$ and $\breve{\mathcal{D}}$, gathered in the following two lemmas. For a proof of the first lemma, see [8, Lemma 2.1]; the second one can be proved by similar arguments. For each radial weight $\omega$ and $x \geqslant 1$, we write $\omega_{x}=\int_{0}^{1} s^{x} \omega(s) d s$.

Lemma B Let $\omega$ be a radial weight. Then the following statements are equivalent:

(i) $\omega \in \widehat{\mathcal{D}}$; 
(ii) There exist constants $C=C(\omega)>0$ and $\beta=\beta(\omega)>0$ such that

$$
\widehat{\omega}(r) \leqslant C\left(\frac{1-r}{1-t}\right)^{\beta} \widehat{\omega}(t), \quad 0 \leqslant r \leqslant t<1 ;
$$

(iii) There exists a constant $C=C(\omega)>0$ such that

$$
\omega_{x} \leqslant C \widehat{\omega}\left(1-\frac{1}{x}\right), \quad 1 \leqslant x<\infty .
$$

Lemma $\mathbf{C}$ Let $\omega$ be a radial weight. Then $\omega \in \check{\mathcal{D}}$ if and only if there exist $C=C(\omega)>0$ and $\gamma=\gamma(\omega)>0$ such that

$$
\widehat{\omega}(t) \leqslant C\left(\frac{1-t}{1-r}\right)^{\gamma} \widehat{\omega}(r), \quad 0 \leqslant r \leqslant t<1 .
$$

Lemma B (ii) shows that if $\omega \in \widehat{\mathcal{D}}$, then there exists $\beta=\beta(\omega)>0$ such that $\frac{\widehat{\omega}(r)}{(1-r)^{\beta}}$ is essentially increasing on $[0,1)$. Similarly, by Lemma $\mathrm{C}, \frac{\widehat{\omega}(r)}{(1-r)^{\gamma}}$ is essentially decreasing on $[0,1)$ for $\gamma=\gamma(\omega)>0$ sufficiently small if $\omega \in \check{\mathcal{D}}$.

\subsection{Necessity}

In this section we prove that $M_{p}(\omega, v, \eta)<\infty$ is a necessary condition for $P_{\omega}^{+}: L_{v}^{p} \rightarrow L_{\eta}^{p}$ to be bounded under the hypotheses of Theorem 1, and establish the desired lower estimate for the operator norm. This is done in the following result under slightly weaker hypotheses than those of the theorem, by using an appropriate family of test functions depending on the weights $\omega$ and $v$.

Proposition 3 Let $1<p<\infty, \omega \in \widehat{\mathcal{D}}$ and $v, \eta$ radial weights. If $P_{\omega}^{+}: L_{v}^{p} \rightarrow L_{\eta}^{p}$ is bounded, then

$$
\sup _{0<r<1}\left(\int_{0}^{r} J_{\omega}(s)^{p} \eta(s) s d s+1\right)^{\frac{1}{p}}\left(\int_{r}^{1}\left(\frac{\omega(s)}{v(s)^{\frac{1}{p}}}\right)^{p^{\prime}} s d s\right)^{\frac{1}{p^{\prime}}} \lesssim\left\|P_{\omega}^{+}\right\|_{L_{\nu}^{p} \rightarrow L_{\eta}^{p}}<\infty,
$$

where $J_{\omega}(s)=\int_{0}^{s} \frac{d t}{\hat{\omega}(t)(1-t)}$ for all $0 \leqslant s<1$.

Proof Assume that $P_{\omega}^{+}: L_{v}^{p} \rightarrow L_{\eta}^{p}$ is bounded, that is,

$$
\int_{\mathbb{D}}\left|\int_{\mathbb{D}} f(\zeta)\right| B_{z}^{\omega}(\zeta)|\omega(\zeta) d A(\zeta)|^{p} \eta(z) d A(z) \leqslant\left\|P_{\omega}^{+}\right\|_{L_{\nu}^{p} \rightarrow L_{\eta}^{p}}^{p}\|f\|_{L_{v}^{p}}^{p}, \quad f \in L_{\nu}^{p},
$$

with $\left\|P_{\omega}^{+}\right\|_{L_{v}^{p} \rightarrow L_{\eta}^{p}}<\infty$. If $v$ vanishes on a set $E \subset \mathbb{D}$ of positive measure, then by choosing $f=\chi_{E}$ the right side of (2.1) is zero. It follows that $\omega$ vanishes (almost everywhere) on $E$ or else $\eta \equiv 0$ (almost everywhere) on $\mathbb{D}$. The latter option being unacceptable as $\hat{\eta}(r)>0$ for all $0 \leqslant r<1$, we deduce that $\omega d A$ is absolutely continuous with respect to $v d A$. Therefore $\omega / v$ is well defined almost everywhere. Hence, for each $n \in \mathbb{N}$ and $0 \leqslant t<1$, the function $f_{n, t}=\min \left\{n,\left(\frac{\omega}{v}\right)^{\frac{1}{p-1}}\right\} \chi \mathbb{D} \backslash D(0, t)$ belongs to $L_{v}^{p}$. A direct calculation shows that 
$f_{n, t}^{p} \nu \leqslant f_{n, t} \omega$ almost everywhere on $\mathbb{D}$. Moreover, by Theorem A (i), there is $r_{0}=r_{0}(\omega)$ such that $M_{1}\left(r, B_{s}^{\omega}\right)=J_{\omega}(r s)$, for any $r, s \geqslant r_{0}$. Hence

$$
M_{1}\left(r, B_{s}^{\omega}\right) \asymp J_{\omega}(r s)+1, \quad 0 \leqslant r, s<1 .
$$

Therefore (2.1) implies

$$
\begin{aligned}
\left\|P_{\omega}^{+}\right\|_{L_{v}^{p} \rightarrow L_{\eta}^{p}}^{p}\left\|f_{n, t}\right\|_{L_{\nu}^{p}}^{p} \gtrsim & \int_{0}^{1}\left(\int_{r}^{1} f_{n, t}(s)\left(J_{\omega}(r s)+1\right) \omega(s) s d s\right)^{p} \eta(r) r d r \\
& +\int_{0}^{1}\left(\int_{0}^{1} f_{n, t}(s) \omega(s) s d s\right)^{p} \eta(r) r d r \\
\geqslant & \int_{0}^{1}\left(\int_{r}^{1} f_{n, t}(s) \omega(s) s d s\right)^{p} \eta(r)\left(J_{\omega}\left(r^{2}\right)+1\right)^{p} r d r \\
& +\left\|f_{n, t}\right\|_{L_{\nu}^{p}}^{p^{2}} \int_{0}^{1} \eta(r) r d r .
\end{aligned}
$$

If we ignore the first summand on the last expression and apply the monotone convergence theorem, we deduce $\int_{0}^{1}\left(\frac{\omega(s)}{v(s)^{\frac{1}{p}}}\right)^{p^{\prime}} s d s<\infty$. This explains why in the statement the supremum over $\left(0, r_{0}\right)$ only, with a prefixed $r_{0} \in(0,1)$, is bounded by a constant $C=C\left(r_{0}\right)>0$ times $\left\|P_{\omega}^{+}\right\|_{L_{v}^{p} \rightarrow L_{\eta}^{p}}$. Further, an application of Lemma B (ii) to $\omega \in \hat{\mathcal{D}}$ gives $J_{\omega}(r)+1 \asymp J_{\omega}\left(r^{2}\right)+1$ for all $0 \leqslant r<1$. Therefore

$$
\begin{aligned}
\left\|P_{\omega}^{+}\right\|_{L_{v}^{p} \rightarrow L_{\eta}^{p}}^{p}\left\|f_{n, t}\right\|_{L_{v}^{p}}^{p} & \gtrsim \int_{0}^{1}\left(\int_{r}^{1} f_{n, t}(s) \omega(s) s d s\right)^{p} \eta(r)\left(J_{\omega}(r)+1\right)^{p} r d r \\
& \geqslant\left(\int_{0}^{t} \eta(r)\left(J_{\omega}(r)+1\right)^{p} r d r\right)\left(\int_{t}^{1} f_{n, t}(s) \omega(s) s d s\right)^{p} \\
& \gtrsim\left(\int_{0}^{t} \eta(r)\left(J_{\omega}(r)+1\right)^{p} r d r\right)\left\|f_{n, t}\right\|_{L_{v}^{p}}^{p^{2}}, \quad n \in \mathbb{N}, \quad 0 \leqslant t<1,
\end{aligned}
$$

because $f_{n, t}^{p} v \leqslant f_{n, t} \omega$ on $\mathbb{D}$. This together with the monotone convergence theorem shows that

$$
\left\|P_{\omega}^{+}\right\|_{L_{v}^{p} \rightarrow L_{\eta}^{p}} \gtrsim\left(\int_{0}^{t} \eta(r)\left(J_{\omega}(r)+1\right)^{p} r d r\right)^{\frac{1}{p}}\left(\int_{t}^{1}\left(\frac{\omega(s)}{v(s)^{\frac{1}{p}}}\right)^{p^{\prime}} s d s\right)^{\frac{1}{p^{\prime}}}, \quad 0<t<1,
$$

and the proposition is proved.

If $\omega \in \widehat{\mathcal{D}}$, then by Lemma B (ii) there exists $\beta(\omega)>0$ such that $J_{\omega}(r) \gtrsim \widehat{\omega}(r)^{-1}(1-$ $(1-r)^{\beta}$ ) for all $0 \leqslant r<1$. Therefore, under the hypotheses of Theorem 1 , we have $\left\|P_{\omega}^{+}\right\|_{L_{v}^{p} \rightarrow L_{\eta}^{p}} \gtrsim M_{p}(\omega, v, \eta)$, and thus the necessity part is proved.

\subsection{Sufficiency}

The proof of the sufficiency of $M_{p}(\omega, v, \eta)<\infty$ for $P_{\omega}^{+}: L_{v}^{p} \rightarrow L_{\eta}^{p}$ to be bounded is more involved than that of the necessity. We begin with the following technical lemma. 
Lemma 4 Let $1<p<\infty$ and $\omega \in \mathcal{D}$ and $v \in \check{\mathcal{D}}$ such that

$$
\int_{0}^{1}\left(\frac{\widehat{\omega}(s)}{\widehat{v}(s)^{\frac{1}{p}}}\right)^{p^{\prime}} \frac{d s}{1-s}<\infty .
$$

Then

$$
\int_{0}^{r}\left(\int_{t}^{1}\left(\frac{\widehat{\omega}(s)}{\hat{v}(s)^{\frac{1}{p}}}\right)^{p^{\prime}} \frac{d s}{1-s}\right)^{\frac{1}{p^{\prime}}} \frac{d t}{\widehat{\omega}(t)(1-t)} \lesssim\left(\int_{r}^{1}\left(\frac{\hat{\omega}(s)}{\widehat{v}(s)^{\frac{1}{p}}}\right)^{p^{\prime}} \frac{d s}{1-s}\right)^{\frac{1}{p^{\prime}}} \frac{1}{\hat{\omega}(r)}, \quad 0 \leqslant r<1 .
$$

Proof Let $\alpha=\alpha(\omega, v, p) \in(0,1)$ to be appropriately fixed later. Then Hölder's inequality and Lemma $\mathrm{C}$ yield

$$
\begin{aligned}
& \int_{0}^{r}\left(\int_{t}^{1}\left(\frac{\widehat{\omega}(s)}{\widehat{v}(s)^{\frac{1}{p}}}\right)^{p^{\prime}} \frac{d s}{1-s}\right)^{\frac{1}{p^{\prime}}} \frac{d t}{\widehat{\omega}(t)(1-t)} \\
& \leqslant\left(\int_{0}^{r}\left(\int_{t}^{1}\left(\frac{\widehat{\omega}(s)}{\widehat{\nu}(s)^{\frac{1}{p}}}\right)^{p^{\prime}} \frac{d s}{1-s}\right) \frac{d t}{\widehat{\omega}(t)^{p^{\prime} \alpha}(1-t)}\right)^{\frac{1}{p^{\prime}}}\left(\int_{0}^{r} \frac{d t}{\widehat{\omega}(t)^{p(1-\alpha)}(1-t)}\right)^{\frac{1}{p}} \\
& \lesssim\left(\int_{0}^{r}\left(\int_{t}^{1}\left(\frac{\widehat{\omega}(s)}{\widehat{v}(s)^{\frac{1}{p}}}\right)^{p^{\prime}} \frac{d s}{1-s}\right) \frac{d t}{\widehat{\omega}(t)^{p^{\prime} \alpha}(1-t)}\right)^{\frac{1}{p^{\prime}}} \frac{1}{\widehat{\omega}(r)^{(1-\alpha)}}, \quad 0 \leqslant r<1,
\end{aligned}
$$

where, by Fubini's theorem and Lemma C,

$$
\begin{aligned}
& \int_{0}^{r}\left(\int_{t}^{1}\left(\frac{\widehat{\omega}(s)}{\widehat{v}(s)^{\frac{1}{p}}}\right)^{p^{\prime}} \frac{d s}{1-s}\right) \frac{d t}{\widehat{\omega}(t)^{p^{\prime} \alpha}(1-t)} \\
& =\int_{0}^{r}\left(\frac{\widehat{\omega}(s)}{\widehat{v}(s)^{\frac{1}{p}}}\right)^{p^{\prime}}\left(\int_{0}^{s} \frac{d t}{\widehat{\omega}(t)^{p^{\prime} \alpha}(1-t)}\right) \frac{d s}{1-s} \\
& +\left(\int_{r}^{1}\left(\frac{\widehat{\omega}(s)}{\widehat{v}(s)^{\frac{1}{p}}}\right)^{p^{\prime}} \frac{d s}{1-s}\right)\left(\int_{0}^{r} \frac{d t}{\widehat{\omega}(t)^{p^{\prime} \alpha}(1-t)}\right) \\
& \lesssim \int_{0}^{r}\left(\frac{\widehat{\omega}(s)^{1-\alpha}}{\widehat{v}(s)^{\frac{1}{p}}}\right)^{p^{\prime}} \frac{d s}{1-s}+\left(\int_{r}^{1}\left(\frac{\widehat{\omega}(s)}{\widehat{v}(s)^{\frac{1}{p}}}\right)^{p^{\prime}} \frac{d s}{1-s}\right) \frac{1}{\widehat{\omega}(r)^{p^{\prime} \alpha}},
\end{aligned}
$$

for all $0 \leqslant r<1$. The latter term is of the desired form. To deal with the first term, observe first that by Lemma C (ii) there exists a constant $\beta=\beta(\omega)>0$ such that $\frac{\hat{\omega}(r)}{(1-r)^{\beta}}$ is essentially increasing on $[0,1)$. Further, for each sufficiently small $\gamma=\gamma(v)>0$ the 
function $\frac{\hat{v}(r)}{(1-r)^{\gamma}}$ is essentially decreasing on $[0,1)$ by Lemma C. Pick up such a $\gamma$ from the interval $(0, p \beta)$, and fix $\alpha \in\left(1-\frac{\gamma}{p \beta}, 1\right)$. Then

$$
\begin{aligned}
\int_{0}^{r}\left(\frac{\widehat{\omega}(s)^{1-\alpha}}{\widehat{v}(s)^{\frac{1}{p}}}\right)^{p^{\prime}} \frac{d s}{1-s} & \lesssim\left(\frac{\widehat{\omega}(r)^{1-\alpha}}{(1-r)^{\beta(1-\alpha)}}\right)^{p^{\prime}} \int_{0}^{r}\left(\frac{(1-s)^{\beta(1-\alpha)}}{\widehat{v}(s)^{\frac{1}{p}}}\right)^{p^{\prime}} \frac{d s}{1-s} \\
& \lesssim\left(\frac{\widehat{\omega}(r)^{1-\alpha}}{(1-r)^{\beta(1-\alpha)}}\right)^{p^{\prime}}\left(\frac{(1-r)^{\frac{\gamma}{p}}}{\widehat{v}(r)^{\frac{1}{p}}}\right)^{p^{\prime}} \int_{0}^{r} \frac{d s}{(1-s)^{1+p^{\prime}\left(\frac{\gamma}{p}-\beta(1-\alpha)\right)}} \\
& \lesssim\left(\frac{\widehat{\omega}(r)^{1-\alpha}}{\widehat{\hat{v}}(r)^{\frac{1}{p}}}\right)^{p^{\prime}} \lesssim\left(\int_{r}^{\frac{1+r}{2}}\left(\frac{\widehat{\omega}(s)}{\widehat{v}(s)^{\frac{1}{p}}}\right)^{p^{\prime}} \frac{d s}{1-s}\right) \frac{1}{\widehat{\omega}(r)^{p^{\prime} \alpha}} \\
& \lesssim\left(\int_{r}^{1}\left(\frac{\widehat{\omega}(s)}{\widehat{v}(s)^{\frac{1}{p}}}\right)^{p^{\prime}} \frac{d s}{1-s}\right) \frac{1}{\widehat{\omega}(r)^{p^{\prime} \alpha}}, \quad 0 \leqslant r<1
\end{aligned}
$$

which together with (2.4) gives

$$
\int_{0}^{r}\left(\int_{t}^{1}\left(\frac{\widehat{\omega}(s)}{\widehat{v}(s)^{\frac{1}{p}}}\right)^{p^{\prime}} \frac{d s}{1-s}\right) \frac{d t}{\widehat{\omega}(t)^{p^{\prime} \alpha}(1-t)} \lesssim\left(\int_{r}^{1}\left(\frac{\widehat{\omega}(s)}{\widehat{v}(s)^{\frac{1}{p}}}\right)^{p^{\prime}} \frac{d s}{1-s}\right) \frac{1}{\widehat{\omega}(r)^{p^{\prime} \alpha}}, \quad 0 \leqslant r<1 .
$$

Finally, by combining the above inequality with (2.3) we obtain the claim.

We are now ready to prove the sufficiency part of Theorem 1 . To do this, assume $M_{p}(\omega, v, \eta)<\infty$, and observe that then the function $h(z)=v(z)^{\frac{1}{p}}$ $\left(\int_{|z|}^{1}\left(\frac{\omega(s)}{v(s)}\right)^{p^{\prime}} v(s) d s\right)^{\frac{1}{p p^{\prime}}}$ is well defined for all $z \in \mathbb{D}$. Hence an integration shows that

$$
\int_{t}^{1}\left(\frac{\omega(s)}{h(s)}\right)^{p^{\prime}} d s=p^{\prime}\left(\int_{t}^{1}\left(\frac{\omega(s)}{v(s)}\right)^{p^{\prime}} v(s) d s\right)^{\frac{1}{p^{\prime}}}, \quad 0 \leqslant t<1 .
$$

Hölder's inequality yields

$$
\begin{aligned}
\left\|P_{\omega}^{+}(f)\right\|_{L_{\eta}^{p}}^{p} \leqslant & \int_{\mathbb{D}}\left(\int_{\mathbb{D}}|f(\zeta)|^{p} h(\zeta)^{p}\left|B_{z}^{\omega}(\zeta)\right| d A(\zeta)\right) \\
& \cdot\left(\int_{\mathbb{D}}\left|B_{z}^{\omega}(\zeta)\right|\left(\frac{\omega(\zeta)}{h(\zeta)}\right)^{p^{\prime}} d A(\zeta)\right)^{p / p^{\prime}} \eta(z) d A(z),
\end{aligned}
$$

where, by (2.2), Fubini's theorem and (2.5),

$$
\begin{aligned}
\int_{\mathbb{D}}\left|B_{z}^{\omega}(\zeta)\right|\left(\frac{\omega(\zeta)}{h(\zeta)}\right)^{p^{\prime}} d A(\zeta) & \lesssim \int_{0}^{1}\left(\frac{\omega(s)}{h(s)}\right)^{p^{\prime}}\left(\int_{0}^{s|z|} \frac{d t}{\hat{\omega}(t)(1-t)}\right) d s+M_{p}(\omega, v, \eta) \\
& =\int_{0}^{|z|}\left(\int_{t /|z|}^{1}\left(\frac{\omega(s)}{h(s)}\right)^{p^{\prime}} d s\right) \frac{d t}{\hat{\omega}(t)(1-t)}+M_{p}(\omega, v, \eta) \\
& \leqslant \int_{0}^{|z|}\left(\int_{t}^{1}\left(\frac{\omega(s)}{h(s)}\right)^{p^{\prime}} d s\right) \frac{d t}{\widehat{\omega}(t)(1-t)}+M_{p}(\omega, v, \eta) \\
& =p^{\prime} \int_{0}^{|z|}\left(\int_{t}^{1}\left(\frac{\omega(s)}{v(s)}\right)^{p^{\prime}} v(s) d s\right)^{\frac{1}{p^{\prime}}} \frac{d t}{\widehat{\omega}(t)(1-t)}+M_{p}(\omega, v, \eta)
\end{aligned}
$$


This together with (2.6), Fubini's theorem and another application of Theorem A (i) gives

$$
\begin{aligned}
\left\|P_{\omega}^{+}(f)\right\|_{L_{\eta}^{p}}^{p} \lesssim & \int_{\mathbb{D}}\left(\int_{\mathbb{D}}|f(\zeta)|^{p} h(\zeta)^{p}\left|B_{z}^{\omega}(\zeta)\right| d A(\zeta)\right) \\
& \cdot\left(\int_{0}^{|z|}\left(\int_{t}^{1}\left(\frac{\omega(s)}{v(s)}\right)^{p^{\prime}} v(s) d s\right)^{\frac{1}{p^{\prime}}} \frac{d t}{\hat{\omega}(t)(1-t)}\right)^{\frac{p}{p^{\prime}}} \eta(z) d A(z) \\
& +M_{p}^{p-1}(\omega, v, \eta) I_{1}(f) \\
\lesssim & \int_{\mathbb{D}}|f(\zeta)|^{p} h(\zeta)^{p}\left[\int_{0}^{1}\left(\int_{0}^{r}\left(\int_{t}^{1}\left(\frac{\omega(s)}{v(s)}\right)^{p^{\prime}} v(s) d s\right)^{\frac{1}{p^{\prime}}} \frac{d t}{\hat{\omega}(t)(1-t)}\right)^{\frac{p}{p^{\prime}}}\right. \\
& \cdot\left(\int_{0}^{r|\zeta|} \frac{d x}{\hat{\omega}(x)(1-x)} \eta(r) r d r\right] d A(\zeta)+M_{p}^{p-1}(\omega, v, \eta) I_{1}(f)+I_{2}(f),
\end{aligned}
$$

where

$$
I_{1}(f)=\int_{\mathbb{D}}\left(\int_{\mathbb{D}}|f(\zeta)|^{p} h(\zeta)^{p}\left|B_{z}^{\omega}(\zeta)\right| d A(\zeta)\right) \eta(z) d A(z)
$$

and

$$
I_{2}(f)=\int_{\mathbb{D}}|f(\zeta)|^{p} h(\zeta)^{p}\left(\int_{0}^{1}\left(\int_{0}^{r}\left(\int_{t}^{1}\left(\frac{\omega(s)}{v(s)}\right)^{p^{\prime}} v(s) d s\right)^{\frac{1}{p^{\prime}}} \frac{d t}{\widehat{\omega}(t)(1-t)}\right)^{p / p^{\prime}} \eta(r) r d r\right) d A(\zeta) .
$$

By (1.4),

$$
\left\|B_{\zeta}^{\omega}\right\|_{A_{\eta}^{1}} \lesssim 1+\int_{0}^{|\zeta|} \frac{\widehat{\eta}(t)}{\widehat{\omega}(t)(1-t)} d t, \quad \zeta \in \mathbb{D} .
$$

This together with Fubini's theorem and Hölder's inequality, implies

$$
\begin{aligned}
I_{1}(f) & =\int_{\mathbb{D}}\left(\int_{\mathbb{D}}|f(\zeta)|^{p} h(\zeta)^{p}\left|B_{z}^{\omega}(\zeta)\right| d A(\zeta)\right) \eta(z) d A(z) \\
& \lesssim \int_{\mathbb{D}}|f(\zeta)|^{p} v(\zeta)\left(\int_{|\zeta|}^{1}\left(\frac{\omega(s)}{v(s)}\right)^{p^{\prime}} v(s) d s\right)^{\frac{1}{p}^{\prime}}\left(\int_{0}^{|\zeta|} \frac{\hat{\eta}(t)}{\hat{\omega}(t)(1-t)} d t\right) d A(\zeta) \\
& +M_{p}(\omega, v, \eta)\|f\|_{L_{v}^{p}}^{p} \\
& \lesssim \int_{\mathbb{D}}|f(\zeta)|^{p} v(\zeta)\left(\int_{|\zeta|}^{1}\left(\frac{\omega(s)}{v(s)}\right)^{p^{\prime}} v(s) d s\right)^{\frac{1}{p^{\prime}}}\left(\int_{0}^{|\zeta|} \frac{\hat{\eta}(t)}{\widehat{\omega}(t)^{p}(1-t)} d t\right)^{\frac{1}{p d A}} \\
& +M_{p}(\omega, v, \eta)\|f\|_{L_{v}^{p}}^{p}
\end{aligned}
$$

because $\widehat{\eta}(\zeta) /(1-|\zeta|)$ is a weight by the hypothesis $\eta \in \breve{\mathcal{D}}$ and Lemma C. Fubini's theorem and Lemma $\mathrm{C}$ for $\omega \in \check{\mathcal{D}}$ give

$$
\int_{0}^{|\zeta|} \frac{\hat{\eta}(t)}{\widehat{\omega}(t)^{p}(1-t)} d t \lesssim \frac{\widehat{\eta}(\zeta)}{\widehat{\omega}(\zeta)^{p}}+\int_{0}^{|\zeta|} \frac{\eta(t)}{\widehat{\omega}(t)^{p}} d t, \quad \zeta \in \mathbb{D} .
$$

But since $\eta \in \check{\mathcal{D}}$ by the hypothesis, there exists a constant $K=K(\eta)>1$ such that $\eta$ satisfies (1.2). Hence, by using Lemma B (ii) for $\omega \in \widehat{\mathcal{D}}$, we deduce

$$
\int_{0}^{r} \frac{\eta(s)}{\widehat{\omega}(s)^{p}} d s \geqslant \int_{1-K(1-r)}^{r} \frac{\eta(s)}{\widehat{\omega}(s)^{p}} d s \geqslant \frac{\int_{1-K(1-r)}^{r} \eta(s) d s}{\widehat{\omega}(1-K(1-r))^{p}} \gtrsim \frac{\widehat{\eta}(r)}{\widehat{\omega}(r)^{p}}, \quad r \geqslant 1-K^{-1},
$$


and hence

$$
\sup _{0<r<1} \frac{\hat{\eta}(r)^{\frac{1}{p}}}{\widehat{\omega}(r)}\left(\int_{r}^{1}\left(\frac{\omega(s)}{v(s)^{\frac{1}{p}}}\right)^{p^{\prime}} d s\right)^{\frac{1}{p^{\prime}}} \lesssim M_{p}(\omega, \nu, \eta)<\infty
$$

It follows that $I_{1}(f) \lesssim M_{p}(\omega, v, \eta)\|f\|_{L_{v}^{p}}^{p}$.

To deal with the remaining terms, we split the integral over $(0,1)$ in $(2.7)$ into two parts at $|\zeta|$. On one hand, since $\omega, \eta \in \breve{\mathcal{D}}$, Lemma $\mathrm{C}$ and (2.10) yield

$$
\begin{aligned}
& \int_{|\zeta|}^{1}\left(\int_{0}^{r}\left(\int_{t}^{1}\left(\frac{\omega(s)}{v(s)}\right)^{p^{\prime}} v(s) d s\right)^{\frac{1}{p^{\prime}}} \frac{d t}{\widehat{\omega}(t)(1-t)}\right)^{p / p^{\prime}}\left(\int_{0}^{r|\zeta|} \frac{d x}{\widehat{\omega}(x)(1-x)}\right) \eta(r) d r \\
& \lesssim \frac{1}{\widehat{\omega}(\zeta)} \int_{|\zeta|}^{1}\left(\int_{0}^{r}\left(\int_{t}^{1}\left(\frac{\omega(s)}{v(s)}\right)^{p^{\prime}} v(s) d s\right)^{\frac{1}{p^{\prime}}} \frac{d t}{\widehat{\omega}(t)(1-t)}\right)^{p / p^{\prime}} \eta(r) d r \\
& \lesssim M_{p}^{\frac{p}{p^{\prime}}}(\omega, v, \eta) \frac{1}{\widehat{\omega}(\zeta)} \int_{|\zeta|}^{1}\left(\int_{0}^{r} \frac{d t}{\widehat{\eta}(t)^{\frac{1}{p}}(1-t)}\right)^{p / p^{\prime}} \eta(r) d r \\
& =M_{p}^{\frac{p}{p^{\prime}}}(\omega, v, \eta) \frac{1}{\widehat{\omega}(\zeta)} \int_{|\zeta|}^{1} \eta(r) \hat{\eta}(r)^{-\frac{1}{p^{\prime}}} d r=M_{p}^{\frac{p}{p^{\prime}}}(\omega, \nu, \eta) p \frac{\hat{\eta}(\zeta)^{\frac{1}{p}}}{\widehat{\omega}(\zeta)}, \quad \zeta \in \mathbb{D} .
\end{aligned}
$$

Therefore, by using (2.10) again we deduce

$$
\begin{aligned}
& h^{p}(\zeta) \int_{|\zeta|}^{1}\left(\int_{0}^{r}\left(\int_{t}^{1}\left(\frac{\omega(s)}{v(s)}\right)^{p^{\prime}} v(s) d s\right)^{\frac{1}{p^{\prime}}} \frac{d t}{\widehat{\omega}(t)(1-t)}\right)^{p / p^{\prime}}\left(\int_{0}^{r|\zeta|} \frac{d x}{\widehat{\omega}(x)(1-x)}\right) \eta(r) r d r \\
& \lesssim M_{p}^{\frac{p}{p^{\prime}}}(\omega, v, \eta) v(\zeta)\left(\int_{|\zeta|}^{1}\left(\frac{\omega(s)}{v(s)}\right)^{p^{\prime}} v(s) d s\right)^{\frac{1}{p^{\prime}}} \frac{\hat{\eta}(\zeta)^{\frac{1}{p}}}{\widehat{\omega}(\zeta)} \lesssim M_{p}^{p}(\omega, \nu, \eta) v(\zeta), \quad \zeta \in \mathbb{D} .
\end{aligned}
$$

On the other hand, since $\omega, v \in \mathcal{R}$, Lemma 4 , Lemma $\mathrm{C}$ and the hypothesis $M_{p}(\omega, v, \eta)<$ $\infty$ yield

$$
\begin{aligned}
& \int_{0}^{|\zeta|}\left(\int_{0}^{r}\left(\int_{t}^{1}\left(\frac{\omega(s)}{v(s)}\right)^{p^{\prime}} v(s) d s\right)^{\frac{1}{p^{\prime}}} \frac{d t}{\widehat{\omega}(t)(1-t)}\right)^{p / p^{\prime}}\left(\int_{0}^{r|\zeta|} \frac{d x}{\widehat{\omega}(x)(1-x)}\right) \eta(r) r d r \\
& \lesssim \int_{0}^{|\zeta|}\left(\int_{r}^{1}\left(\frac{\omega(s)}{v(s)}\right)^{p^{\prime}} v(s) d s\right)^{\frac{p}{\left.p^{\prime}\right)^{2}}} \frac{1}{\widehat{\omega}(r)^{p-1}}\left(\int_{0}^{r} \frac{d x}{\widehat{\omega}(x)(1-x)}\right) \eta(r) d r \\
& \lesssim M_{p}^{\frac{p}{p^{\prime}}}(\omega, \nu, \eta) \int_{0}^{|\zeta|} \frac{\eta(r)}{\widehat{\omega}(r)^{p}\left(\int_{0}^{r} \frac{\eta(s)}{\hat{\omega}(s)^{p}} d s\right)^{\frac{1}{p^{\prime}}}} d r=M_{p}^{\frac{p}{p^{\prime}}}(\omega, \nu, \eta)\left(\int_{0}^{|\zeta|} \frac{\eta(s)}{\widehat{\omega}(s)^{p}} d s\right)^{\frac{1}{p}}, \quad \zeta \in \mathbb{D} .
\end{aligned}
$$


This together with the hypothesis $M_{p}(\omega, v, \eta)<\infty$ gives

$$
\begin{aligned}
& h^{p}(\zeta) \int_{0}^{|\zeta|}\left(\int_{0}^{r}\left(\int_{t}^{1}\left(\frac{\omega(s)}{v(s)}\right)^{p^{\prime}} v(t) d t\right)^{\frac{1}{p^{\prime}}} \frac{d t}{\hat{\omega}(t)(1-t)}\right)^{p / p^{\prime}}\left(\int_{0}^{r|\zeta|} \frac{d x}{\widehat{\omega}(x)(1-x)}\right) \eta(r) r d r \\
& \lesssim M_{p}^{\frac{p}{p^{\prime}}}(\omega, \nu, \eta) v(\zeta)\left(\int_{|\zeta|}^{1}\left(\frac{\omega(s)}{v(s)}\right)^{p^{\prime}} v(s) d s\right)^{\frac{1}{p^{\prime}}}\left(\int_{0}^{|\zeta|} \frac{\eta(s)}{\widehat{\omega}(s)^{p}} d s\right)^{\frac{1}{p}} \lesssim M_{p}^{p}(\omega, \nu, \eta) v(\zeta), \quad \zeta \in \mathbb{D} .
\end{aligned}
$$

Consequently, by combining the previous estimates, we deduce that the third to last term in (2.7) is bounded by a constant times $M_{p}^{p}(\omega, v, \eta)\|f\|_{L_{v}^{p}}^{p}$. In order to bound $I_{2}(f)$, let us observe that that the third to last term in (2.7) differs from $I_{2}(f)$ only by the extra factor $\left(\int_{0}^{r|\zeta|} \frac{d x}{\hat{\omega}(x)(1-x)}\right)$, which has been bounded by a constant times $\max \left\{\frac{1}{\hat{\omega}(r)}, \frac{1}{\hat{\omega}(|\zeta|)}\right\}$ in the calculations above. Since $\max \left\{\frac{1}{\hat{\omega}(r)}, \frac{1}{\hat{\omega}(|\zeta|)}\right\}$ is uniformly bounded away from zero on $\mathbb{D}$, the same reasoning shows that $I_{2}(f) \lesssim M_{p}^{p}(\omega, v, \eta)\|f\|_{L_{v}^{p}}^{p}$. These inequalities, together with (2.7) and (2.9) give $\left\|P_{\omega}^{+}(f)\right\|_{L_{\eta}^{p}} \lesssim M_{p}(\omega, v, \eta)\|f\|_{L_{v}^{p}}$ as claimed.

\section{Proof of Theorem 2}

To prove Theorem 2, we first show that $N_{p}(\omega, v, \eta) \lesssim\left\|P_{\omega}\right\|_{L_{v}^{p} \rightarrow L_{\eta}^{p}}$ under the hypotheses of the theorem.

Proposition 5 Let $1<p<\infty, \omega \in \widehat{\mathcal{D}}, \eta \in \mathcal{D}$ and $v$ a radial weight. If $P_{\omega}: L_{v}^{p} \rightarrow L_{\eta}^{p}$ is bounded, then

$$
\sup _{0<r<1} \frac{\hat{\eta}(r)^{\frac{1}{p}}}{\widehat{\omega}(r)}\left(\int_{r}^{1}\left(\frac{\omega(t)}{v(t)}\right)^{p^{\prime}} v(t) t d t\right)^{\frac{1}{p^{\prime}}} \lesssim\left\|P_{\omega}\right\|_{L_{v}^{p} \rightarrow L_{\eta}^{p}} .
$$

Proof The adjoint of $P_{\omega}$ is defined by

$$
\left\langle P_{\omega}(f), g\right\rangle_{L_{\eta}^{2}}=\left\langle f, P_{\omega}^{\star}(g)\right\rangle_{L_{v}^{2}}, \quad f \in L_{v}^{p}, \quad g \in L_{\eta}^{p^{\prime}} .
$$

Now [10, Theorem 1(i)] and Lemma $\mathrm{C}$, applied to $\eta \in \check{\mathcal{D}}$, yield

$$
\int_{\mathbb{D}}\left(\int_{\mathbb{D}}\left|B_{\zeta}^{\omega}(z)\right| \omega(\zeta) d A(\zeta)\right) \eta(z) d A(z) \lesssim \int_{0}^{1} \eta(r) \log \frac{e}{1-r} d r=\int_{0}^{1} \frac{\hat{\eta}(r)}{1-r} d r<\infty .
$$

If $f$ and $g$ are bounded functions, then (3.1) shows that we may apply Fubini's theorem to deduce

$$
\begin{aligned}
\left\langle P_{\omega}(f), g\right\rangle_{L_{\eta}^{2}} & =\int_{\mathbb{D}} P_{\omega}(f)(z) \overline{g(z)} \eta(z) d A(z) \\
& =\int_{\mathbb{D}}\left(\int_{\mathbb{D}} f(\zeta) B_{\zeta}^{\omega}(z) \omega(\zeta) d A(\zeta)\right) \overline{g(z)} \eta(z) d A(z) \\
& =\int_{\mathbb{D}} f(\zeta)\left(\int_{\mathbb{D}} \overline{g(z)} B_{\zeta}^{\omega}(z) \eta(z) d A(z)\right) \omega(\zeta) d A(\zeta) \\
& =\int_{\mathbb{D}} f(\zeta) \overline{\left(\frac{\omega(\zeta)}{v(\zeta)} \int_{\mathbb{D}} g(z) B_{z}^{\omega}(\zeta) \eta(z) d A(z)\right)} v(\zeta) d A(\zeta)=\left\langle f, P_{\omega}^{\star}(g)\right\rangle_{L_{v}^{2}} .
\end{aligned}
$$


Since the simple functions are dense in $L_{\sigma}^{p}$ for each $1 \leqslant p<\infty$ and radial $\sigma$, this shows that

$$
P_{\omega}^{\star}(g)(\zeta)=\frac{\omega(\zeta)}{v(\zeta)} \int_{\mathbb{D}} g(z) B_{z}^{\omega}(\zeta) \eta(z) d A(z), \quad \zeta \in \mathbb{D}, \quad g \in L_{\eta}^{p^{\prime}}
$$

The adjoint operator $P_{\omega}^{\star}: L_{\eta}^{p^{\prime}} \rightarrow L_{v}^{p^{\prime}}$ is bounded by the hypothesis, and $\left\|P_{\omega}^{\star}\right\|_{L_{\eta}^{p^{\prime}} \rightarrow L_{v}^{p^{\prime}}}=$ $\left\|P_{\omega}\right\|_{L_{v}^{p} \rightarrow L_{\eta}^{p}}$. Thus

$$
\int_{\mathbb{D}}\left(\frac{\omega(\zeta)}{v(\zeta)}\right)^{p^{\prime}}\left|\int_{\mathbb{D}} g(z) B_{z}^{\omega}(\zeta) \eta(z) d A(z)\right|^{p^{\prime}} v(\zeta) d A(\zeta) \leqslant\left\|P_{\omega}\right\|_{L_{\nu}^{p} \rightarrow L_{\eta}^{p}}^{p^{\prime}}\|g\|_{L_{\eta}^{p^{\prime}}}^{p^{\prime}}, \quad g \in L_{\eta}^{p^{\prime}}
$$

By considering the standard orthonormal basis $\left\{z^{j} / \sqrt{2 \omega_{2 j+1}}\right\}, j \in \mathbb{N} \cup\{0\}$, of the Hilbert space $A_{\omega}^{2}$, one deduces

$$
B_{z}^{\omega}(\zeta)=\sum_{n=0}^{\infty} \frac{(\zeta \bar{z})^{n}}{2 \omega_{2 n+1}}, \quad z, \zeta \in \mathbb{D}
$$

By testing (3.2) with monomials $g_{n}(\zeta)=\zeta^{n}$ we obtain

$$
\begin{aligned}
\left(\frac{\eta_{2 n+1}}{\omega_{2 n+1}}\right)^{p^{\prime}} \int_{\mathbb{D}}|\zeta|^{n p^{\prime}}\left(\frac{\omega(\zeta)}{v(\zeta)}\right)^{p^{\prime}} v(\zeta) d A(\zeta) & =\left\|P_{\omega}^{\star}\left(g_{n}\right)\right\|_{L_{\eta}^{p^{\prime}}}^{p^{\prime}} \leqslant\left\|P_{\omega}\right\|_{L_{\nu}^{p} \rightarrow L_{\eta}^{p}}^{p^{\prime}}\left\|g_{n}\right\|_{L_{\eta}^{p^{\prime}}}^{p^{\prime}} \\
& =2\left\|P_{\omega}\right\|_{L_{\nu}^{p} \rightarrow L_{\eta}^{p}}^{p^{\prime}} \eta_{n p^{\prime}+1}, \quad n \in \mathbb{N} \cup\{0\},
\end{aligned}
$$

from which Lemma B(ii)(iii), applied to $\omega, \eta \in \widehat{\mathcal{D}}$, yields

$$
\begin{aligned}
\left\|P_{\omega}\right\|_{L_{v}^{p} \rightarrow L_{\eta}^{p}}^{p^{\prime}} & \gtrsim \sup _{n \in \mathbb{N}}\left(\frac{\eta_{2 n+1}^{p^{\prime}}}{\omega_{2 n+1}^{p^{\prime}} \eta_{n p^{\prime}+1}} \int_{0}^{1} t^{n p^{\prime}}\left(\frac{\omega(t)}{v(t)}\right)^{p^{\prime}} v(t) t d t\right) \\
& \approx \sup _{n \in \mathbb{N}}\left(\frac{\widehat{\eta}\left(1-\frac{1}{n}\right)^{p^{\prime}-1}}{\widehat{\omega}\left(1-\frac{1}{n}\right)^{p^{\prime}}} \int_{0}^{1} t^{n p^{\prime}}\left(\frac{\omega(t)}{v(t)}\right)^{p^{\prime}} v(t) t d t\right) \\
& \gtrsim \sup _{n \in \mathbb{N} \backslash\{1\}}\left(\frac{\widehat{\eta}\left(1-\frac{1}{n}\right)^{p^{\prime}-1}}{\widehat{\omega}\left(1-\frac{1}{n}\right)^{p^{\prime}}} \int_{1-\frac{1}{n}}^{1}\left(\frac{\omega(t)}{v(t)}\right)^{p^{\prime}} v(t) t d t\right) .
\end{aligned}
$$

Let $\frac{1}{2} \leqslant r<1$ and fix $n \in \mathbb{N}$ such that $1-\frac{1}{n} \leqslant r<1-\frac{1}{n+1}$. By applying Lemma B (ii) again we finally deduce

$$
\left\|P_{\omega}\right\|_{L_{\nu}^{p} \rightarrow L_{\eta}^{p}} \gtrsim \frac{\widehat{\eta}(r)^{\frac{1}{p}}}{\widehat{\omega}(r)}\left(\int_{r}^{1}\left(\frac{\omega(t)}{v(t)}\right)^{p^{\prime}} v(t) t d t\right)^{\frac{1}{p^{\prime}}}, \quad \frac{1}{2} \leqslant r<1 .
$$

The assertion follows from this inequality because the last integral converges for $r=0$ by (3.3) with $n=0$. 
Proof of Theorem 2. By Theorem 1 and Proposition 5, it suffices to show that (iii) implies (iv) under the hypothesis (1.5). To see this, first observe that an integration by parts and Hölder's inequality give

$$
\begin{aligned}
\int_{0}^{r} \frac{\eta(s)}{\widehat{\omega}(s)^{p}} d s & \leqslant \frac{\widehat{\eta}(0)}{\widehat{\omega}(0)^{p}}+p \int_{0}^{r} \frac{\widehat{\eta}(s) \omega(s)}{\widehat{\omega}(s)^{p+1}} d s \\
& =\frac{\widehat{\eta}(0)}{\widehat{\omega}(0)^{p}}+p \int_{0}^{r} \frac{\widehat{\eta}(s)}{\widehat{\omega}(s)^{p}} \frac{\omega(s)}{\eta(s)^{\frac{1}{p}}} \frac{\eta(s)^{\frac{1}{p}}}{\widehat{\omega}(s)} d s \\
& \leqslant \frac{\widehat{\eta}(0)}{\widehat{\omega}(0)^{p}}+p\left(\int_{0}^{r}\left(\frac{\hat{\eta}(s)}{\widehat{\omega}(s)^{p}} \frac{\omega(s)}{\eta(s)^{\frac{1}{p}}}\right)^{p^{\prime}} d s\right)^{\frac{1}{p^{\prime}}}\left(\int_{0}^{r} \frac{\eta(s)}{\widehat{\omega}(s)^{p}} d s\right)^{\frac{1}{p}}
\end{aligned}
$$

Moreover, since $N_{p}(\omega, v, \eta)<\infty$, we have

$$
\begin{aligned}
& \left(\int_{0}^{r}\left(\frac{\widehat{\eta}(s)}{\widehat{\omega}(s)^{p}} \frac{\omega(s)}{\eta(s)^{\frac{1}{p}}}\right)^{p^{\prime}} d s\right)^{\frac{1}{p^{\prime}}} \\
\leqslant & N_{p}^{p}(\omega, v, \eta)\left(\int_{0}^{r}\left(\frac{\omega(s)}{\eta(s)^{\frac{1}{p}}}\right)^{p^{\prime}}\left(\int_{s}^{1}\left(\frac{\omega(t)}{v(t)^{\frac{1}{p}}}\right)^{p^{\prime}} d t\right)^{-p} d s\right)^{\frac{1}{p^{\prime}}} .
\end{aligned}
$$

By combining (3.4) and (3.5), we deduce

$$
\begin{aligned}
& \int_{0}^{r} \frac{\eta(s)}{\widehat{\omega}(s)^{p}} d s \\
& \leqslant \frac{\widehat{\eta}(0)}{\hat{\omega}(0)^{p}}+p N_{p}^{p}(\omega, v, \eta)\left(\int_{0}^{r}\left(\frac{\omega(s)}{\eta(s)^{\frac{1}{p}}}\right)^{p^{\prime}}\left(\int_{s}^{1}\left(\frac{\omega(t)}{v(t)^{\frac{1}{p}}}\right)^{p^{\prime}} d t\right)^{-p} d s\right)^{\frac{1}{p^{\prime}}}\left(\int_{0}^{r} \frac{\eta(s)}{\widehat{\omega}(s)^{p}} d s\right)^{\frac{1}{p}} .
\end{aligned}
$$

Therefore

$$
\left(\int_{0}^{r} \frac{\eta(s)}{\widehat{\omega}(s)^{p}} d s\right)^{\frac{1}{p}} \lesssim N_{p}^{p^{\prime}}(\omega, v, \eta)\left(\int_{0}^{r}\left(\frac{\omega(s)}{\eta(s)^{\frac{1}{p}}}\right)^{p^{\prime}}\left(\int_{s}^{1}\left(\frac{\omega(t)}{v(t)^{\frac{1}{p}}}\right)^{p^{\prime}} d t\right)^{-p} d s\right)^{\frac{1}{p}}
$$

for all $\frac{1}{2} \leqslant r<1$. This together with the hypothesis (1.5) completes the proof.

Acknowledgement The authors would like to thank the referee for careful reading of the manuscript and for pointing out a matter which yielded the final version of Theorem 2 .

Funding Information Open access funding provided by University of Eastern Finland (UEF) including Kuopio University Hospital.

Open Access This article is licensed under a Creative Commons Attribution 4.0 International License, which permits use, sharing, adaptation, distribution and reproduction in any medium or format, as long as you give appropriate credit to the original author(s) and the source, provide a link to the Creative Commons licence, and indicate if changes were made. The images or other third party material in this article are included in the article's Creative Commons licence, unless indicated otherwise in a credit line to the material. If material is not included in the article's Creative Commons licence and your intended use is not permitted by statutory regulation or exceeds the permitted use, you will need to obtain permission directly from the copyright holder. To view a copy of this licence, visit http://creativecommonshorg/licenses/by/4.0/. 


\section{References}

1. Aleman, A., Constantin, O.: The Bergman projection on vector-valued $l^{2}$-spaces with operator-valued weights. J. Funct. Anal. 262(5), 2359-2378 (2012)

2. Aleman, A., Pott, S., Reguera, M.C.: Sarason conjecture on the Bergman space. Int. Math. Res. Not. IMRN 14, 4320-4349 (2017)

3. Bekollé, D.: Inégalités á poids pour le projecteur de Bergman dans la boule unité de $\mathbb{C}^{n}$ [Weighted inequalities for the Bergman projection in the unit ball of $\mathbb{C}^{n}$ ]. Studia Math. 71(3), 305-323 (1981/82)

4. Bekollé, D., Bonami, A.: Inégalités á poids pour le noyau de Bergman, (French) C. R. Acad. Sci. Paris Sr A-B 286(18), 775-778 (1978)

5. Hytönen, T.: The sharp weighted bound for general calderón-zygmund operators. Ann. of Math. (2) 175(3), 1473-1506 (2012)

6. Lacey, M., Sawyer, E., Chun-Yen, S., Uriarte-Tuero, I.: Two-weight inequality for the Hilbert transform: a real variable characterization. I. Duke Math. J. 163(15), 2795-2820 (2014)

7. Lacey, M., Sawyer, E., Chun-Yen, S., Uriarte-Tuero, I., Wick, B.D.: Two weight inequalities for the cauchy transform from $\mathbb{R}$ to $\mathbb{C}^{+}$, arXiv: 1310.4820

8. Peláez, J.A.: Small weighted Bergman spaces Proceedings of the summer school in complex and harmonic analysis, and related topics (2016)

9. Peláez, J., Rättyä, J.: Weighted Bergman spaces induced by rapidly increasing weights, Mem. Amer. Math. Soc. 227 (2014)

10. Peláez, J.A., Rättyä, J.: Two weight inequality for Bergman projection, J. Math. Pures Appl. (9)105 no 1, 102-130 (2016)

11. Peláez, J.A., Rättyä, J., Wick, B.D.: Bergman projection induced by kernel with integral representation. J. Anal. Math. 138(1), 325-360 (2019)

12. Peláez, J.A., Rättyä, J.: Bergman projection induced by radial weight, preprint.

13. Pott, S., Reguera, M.C.: Sharp bekollé estimate for the Bergman projection. J. Funct. Anal. 265, 3233 3244 (2013)

Publisher's Note Springer Nature remains neutral with regard to jurisdictional claims in published maps and institutional affiliations. 